Case 2 - Five-year old boy, has acquired microcephaly since 12 months of age, absent speech, mental retardation, behavioral problems. Creatine Transport Defect was confirmed with high levels of creatine in urine and hemizygous missense mutation in the SLC6A8 gene.

Case 3 - Eleven-months old boy, has acquired microcephaly since six months of age, absent speech, hypotonia. He also has $\alpha$ 1-antitrypsin deficiency, increased lactic acid and T4 low. Cerebral magnetic resonance showed global loss of volume of white matter. Muscle biopsy confirmed respiratory chain disorder with complex 2 deficiency - $25 \%$.

Discussion If the patient showed acquired microcephaly and absent speech associated with convulsions and angelman-like features, the most probably diagnosed is Angelman syndrome. The screening for mutation in chromosome 15 diagnosed the syndrome. If the patient has also behavior disturbances with family history of learning disabilities, determination of urine creatine is obligatory to exclude creatine transport defect. If all these tests are negative and the patient has unrelated organs involved, we need to exclude respiratory chain disorder and muscle biopsy is mandatory.

\section{CLINICAL AND THERAPEUTIC ASPECTS OF GAUCHER'S DISEASE IN CHILDREN}

doi:10.1136/archdischild-2012-302724.0531

A Benketira. Pediatric, Military Hospital Regional University of Oran, Oran, Algeria

Introduction Gaucher's disease is the most common of the inherited metabolic disorder known as lipid storage diseases. It is a lysosomal disease, autosomal recessive. It is caused by a deficiency of beta-glucocerebrosidase. The result is a substance called glucocerebroside to build up in cells of the body (Spleen, liver, lungs, bones and sometimes in the brain.

There are three clinicals types:

Type 1

$95 \%$

$1 / 50000$

Subacute

Infants/Children

Doesn't involve the brain

Type 2

$1 \%$

$1 / 150000$

Acute/Deadly

Newborn-06 months

Severe brain damage

Type 3;

$5 \%$

$1 / 100000$

Chronic

Juvenil/Adult

Brain-Liver-Spleen involvement appear gradually

Materials and Methods It's a baby 13 months old. He had hepatosplenomegaly with cytopenia. He had the neurological signs such pyramidal syndrome with contra version ocular without flutter.

The exploration concluded for the GD by the enzymatic dosage.

Results After six years of follow up, enzyme replacement therapy (Imiglucerase) has demonstrated its effectiveness as well as biological as clinical.

Our observation has been raised the possibility of signs of brain involvement in the type 1 .

The finding joins a few cases in the literature.

This data calls into question the traditional classification cited from above.
Conclusion We emphasize the importance of studying more of cases (Clinic - Genetic) to put an update on the current classification.

The early therapeutic in the management of GD is still advantageous.

\section{VACTERL ASSOCIATION: A NEW CASE WITH BIOTINIDASE DEFICIENCY AND ANNULAR PANCREAS}

doi:10.1136/archdischild-2012-302724.0532

${ }^{1} \mathrm{RG}$ Sezer, ${ }^{2} \mathrm{G}$ Aydemir, ${ }^{1} \mathrm{~A}$ Bozaykut, ${ }^{1} \mathrm{C}$ Paketci, ${ }^{2} \mathrm{~S}$ Aydinoz. ${ }^{1}$ Department of Pediatrics, Zeynep Kamil Maternity and Childrens' Diseases Training and Research State Hospital; 2Pediatrics, GATA Haydarpasa Teaching Hospital, Istanbul, Turkey

VACTERL (V - Vertebral anomalies, A - Anal atresia, C - Cardiovascular anomalies, T - Tracheoesophageal fistula, E - Esophageal atresia, R - Renal (Kidney) and defects, anal atresia, cardiac defects, tracheo-esophageal fistula, renal dysplasia, and limb anomalies. Less frequent defects seen with VACTERL association are prenatal and postnatal growth deficiency, laryngeal stenosis, ear anomaly, large fontanels, defect of lower limb, rib anomaly, tethered cord, and defects of external genitalia. We report a case of VACTERL association who had concomitant biotinidase deficiency and annular pancreas, which has not been previously reported.

\section{CANAVAN DISEASE: A CASE REPORT FROM KUWAIT}

doi:10.1136/archdischild-2012-302724.0533

HA Bin Nakhi, A Ipe Jhon, R Pinto. Pediatrics, Adan Hospital, Kuwait, Kuwait

A Kuwaiti female presented at age of three months with macrocephaly, hypotonia, and global developemental delay. She was found to have Cancvan disease. Although Cancvan disease is a rare autosomal recessive neurometabolic disorder which occurs mainly in Ashkanazi Jews, we are reporting this case to highlight that neurometabolic diseases as well as other rare autosomal-recessive disorders affect a relatively large number of patients in countries with high rate of consanguineous marrige like Kuwait and other Gulf areas. We believe that it is high time for molecular cytogenetic studies to be done on Canvan disease and other rare neurometabolic disorders affecting Kuwaiti patients.

\section{A CHALLENGING CASE OF MAKING CRITICAL CARE DECISION ON THE WITHDRAWAL OF NEONATAL INTENSIVE CARE}

doi:10.1136/archdischild-2012-302724.0534

A Singh, SV Rasiah. Neonatal Intensive Care Unit, Birmingham Women's NHS Foundation Trust, Birmingham, UK

Background The RCPCH and the Nuffield Council of Bioethics provide guidance on making critical care decisions on withdrawal of intensive care. BLISS also provides guidance for families to work in partnership with professionals when making critical decisions.

Clinical Case Following an uneventful pregnancy, a baby boy was born at 30 weeks gestation to non-consanguineous parents. He was profoundly hypotonic with respiratory distress at birth. $\mathrm{He}$ remained ventilated for poor respiratory effort in the weeks following delivery. He failed attempts at extubation. He continued to have paucity of movement. He underwent extensive investigations for hypotonia and was reviewed by external specialists. His clinical picture suggested an extremely poor prognosis. Following extensive discussions and multidisciplinary meetings it was felt that it was not in the baby's best interest to continue with intensive care. Despite prolonged counselling of the parents over weeks regarding palliative care, they insisted on continuing intensive care. They sought an independent neonatal opinion through their solicitor. The opinion of the independent external professional was in 
agreement with our assessment. Genetic testing was subsequently positive for congenital myotonic dystrophy. Parents finally consented to withdrawal of intensive care at day 64 of life and he died shortly after extubation.

Conclusion Critical care decision on withdrawal of intensive care can be a very traumatic experience for families. It is essential to follow the guidance available. As paediatricians we are advocates for the baby but at the same time we have to be empathetic and considerate to the sentiments of the family.

\section{NECROTIZING ENTEROCOLITIS IN A NEWBORN FOLLOWING INTRAVENOUS IMMUNGLOBULIN TREATMENT FOR HEMOLYTIC DISEASE}

doi:10.1136/archdischild-2012-302724.0535

'S Kara, ${ }^{2} \mathrm{H}$ Ulu-Özkan, ${ }^{2 Y}$ Y YImaz, ${ }^{3} \mathrm{Fi}$ Arıkan, ${ }^{2} \mathrm{U}$ Dilmen, ${ }^{3} \mathrm{Y}$ Dallar Bilge. ${ }^{1}$ Ankara Training and Research Hospital, Department of Neonatology; 'Zekai Tahir Burak Maternity Training Hospital, Department of Neonatology: ${ }^{3}$ Ankara Training and Research Hospital, Department of Pediatrics, Ankara, Turkey

$\mathrm{ABO}$ iso-immunization is the most frequent hemolytic disease of the newborn. Treatment depends on the total serum bilirubin level, which may increase very rapidly in the first $48 \mathrm{~h}$ of life in cases of hemolytic disease of the newborn. Phototherapy and, in severe cases, exchange transfusion are used to prevent hyperbilirubinemia encephalopathy. Intravenous immunoglobulins are used to reduce exchange transfusion. Herein we present a female infant who was admitted to the our NICU because of ABO immune hemolytic disease and after two courses of $1 \mathrm{gr} / \mathrm{kg}$ of IVIG infusion, she developed NEC. Administration of IVIG to newborns with significant hyperbilirubinemia due to $\mathrm{ABO}$ hemolytic disease should be cautiously employed and always administered under strict.

\section{NEONATAL HSV ENCEPHALITIS: CONTROVERSIES OVER DIFFERENT THERAPEUTIC APPROACHES AND THEIR EFFECTS ON NEURO-DEVELOPMENTAL OUTCOMES}

doi:10.1136/archdischild-2012-302724.0536

A Papandreou, E Lek, A Pahuja, C Ramesh. Paediatrics, West Hertfordshire Hospitals NHS Trust, Watford, UK

Background and Aims Neonatal HSV encephalitis is well described and known to cause morbidity. However, there is no consensus regarding its optimal treatment, especially around using suppressive oral therapy after intravenous acyclovir. We aim to discuss treatment controversies and review possible neurodevelopmental outcomes in such cases.

Methods We report a case of vertically transmitted neonatal HSV-1 encephalitis and review existing literature on available treatment options (PubMed, EMBASE).

Results Our patient became pyrexial (39.0C) and lethargic on day 7 of life. Investigations revealed a raised CRP $(80 \mathrm{mg} / \mathrm{l})$ and CSF pleocytosis (WCC-26/ $\mathrm{mm}^{3}$, 90\% lymphocytes) with normal CSF biochemistry. IV antibiotics were empirically started. After developing encephalopathy and seizures on day 2 of illness, IV acyclovir was added. CSF PCR was positive for HSV-1. EEG showed multifocal irritability/excitability and asymmetrical temporal lobe activities. MRI showed low signal intensity on the ADC map in the medial temporal lobe cortices bilaterally and the right inferior frontal cortex.

21 days of IV acyclovir were completed, following which a repeat CSF sample was negative for HSV-1 PCR. IV antivirals were substituted with oral acyclovir at $1500 \mathrm{mg} / \mathrm{m}^{2} /$ dose BD for twelve months. Conclusions Literature review reveals controversies in treatment. Repeating HSV PCR at the end of IV treatment is not universally supported. Regarding suppressive oral acyclovir, some studies support doses of $1000-1740 \mathrm{mg} / \mathrm{m}^{2} /$ dose BD while others favour a
$300 \mathrm{mg} / \mathrm{m}^{2} /$ dose TDS regime. Its optimal duration (6months, 12 months or longer) is unclear. Neurodevelopmental outcomes mostly depend on the severity of the initial insult; Evidence that different suppressive treatments influence outcomes is poor.

\section{AN IMPORTANT CAUSE OF DYSKINESIA}

doi:10.1136/archdischild-2012-302724.0537

C Peevers. Paediatric Neurology, Bristol Children's Hospital, Bristol, UK

Kernicterus has been referred to a disease of the past. However, we report two cases of kernicterus presenting with a dyskinetic movement disorder. Both cases had neonatal jaundice and were well until the age of 3 years with normal intellect. On examination dystonia, dyskinesia and chorea were seen. Further examination revealed an upgaze palsy and auditory neuropathy.

Kernicterus describes a neurological syndrome resulting from deposition of unconjuated bilirubin in basal ganglia \& brainstem nuclei.

With the recent NICE guidance for jaundice therapy these cases highlight the importance of rigorous treatment of hyperbilirubinaemia. They also remind us to consider kernicterus as a diagnosis in a child presenting with a movement disorder and normal intellect.

\section{THE VALUE OF NEAR-INFRARED SPECTROSCOPY (NIRS) IN PERINATAL ASPHYXIA-A CASE REPORT}

doi:10.1136/archdischild-2012-302724.0538

${ }^{1} \mathrm{~F}$ Norooz, ${ }^{2 B}$ Urlesberger, ${ }^{1} \mathrm{~K}$ Klebermasz-Schrehof, ' $\mathrm{C}$ Czaba, ${ }^{1} \mathrm{E}$ Obwegeser, 'V Giordano, 'A Berger, 'M Weninger, 'M Olischar. 'Division of Paediatric Neonatology, Intensive Care and Neuropaediatrics, Medical University of Vienna, Vienna; ${ }^{2}$ Department of Paediatrics and Adolescent Medicine, Division of Neonatology, Medical University of Graz, Graz, Austria

Background Perinatal asphyxia remains a challenging entity. NIRS offers a method to continuously monitor cerebral oxygen saturation.

Aim To obtain insight into haemodynamic changes during hypothermia and rewarming in perinatal asphyxia using NIRS.

Methods We report of an asphyxiated patient $(37+6$ weeks', Apgar 6 and 8 at 1 and 5 minutes, first arterial blood gas $\mathrm{pH}$ of 6.67 , base deficit -25). NIRS was started during the first hour of life and continued for a total recording time of 125 hours. Simultaneously, we measured brain function using amplitude-integrated electroencephalography (aEEG). On day 7 magnetic resonance imaging (MRI) has been performed. After discharge, the patient was reassessed neurologically.

Results The initial cerebral $\mathrm{rSO} 2$ was $65 \%$. When cooling was started FTOE was 0.28 . At $33.5^{\circ} \mathrm{C}$ FTOE had decreased to 0.20 , cerebral $\mathrm{rSO} 2$ increased to $70 \%$. After rewarming cerebral $\mathrm{rSO} 2$ was $85 \%$, and FTOE 0.11. Initially, aEEG showed a mixed burst-suppression and discontinuous pattern which improved to a discontinuous pattern only during the first 12 hours. After rewarming aEEG normalized and showed developing sleep-wake cycles. MRI did not show any signs of hypoxic damage. After discharge the patient presented neurodevelopmentally normal.

Conclusion After having cooled down the patient, both NIRS and aEEG showed an improvement (increase of rSO2, decrease of FTOE, loss of burst-supression in aEEG). aEEG displays cerebral function, cerebral NIRS expands information to cerebral oxygen supply and extraction. MRI and neurodevelopmental assessment proved the observed aEEG and NIRS data.

539 SEVERE FORM OF CONGENITAL TOXOPLASMOSIS WITH EXTENSIVE CEREBRAL FINDINGS

doi:10.1136/archdischild-2012-302724.0539 